\title{
IS THE GEODESIC HYPOTHESIS IN GENERAL RELATIVITY FALSIFIABLE?
}

\author{
STANISŁAW L. BAŻAŃSKI \\ Institute of Theoretical Physics, University of Warsaw \\ ul. Hoża 69, 00-681 Warszawa, Poland \\ E-mail:bazanski@fuw.edu.pl
}

I. Introduction. It is generally accepted that a theoretical result is tested in an experiment if the numerical data implied by such a result can be reproduced, usually with a certain accuracy, in consequence of a number of suitably designed experimental measurements. So long experimental physicists cannot perform any measurements that disagree with the implications of the theoretical result in question, the theory on the basis of which the result has been derived is regarded as being experimentally verified with an increased degree of confidence.

The scheme of gradually adding, in confrontation with the experiment, new theoretical information to the knowledge is in accordance with the inductive method first introduced to modern science by Sir Francis Bacon in his Advancement of Learning and Novum Organum. This method was later developed to a more sophisticated stage by generations of scholars. The key figures who contributed mainly to this development were David Hume, John Stuart Mill, and more recently Rudolf Carnap and numerous other representatives of the neopositivist philosophy who have strongly influenced views on contemporary physics, held at least in some circles. An adherent of this philosophy who to a certain degree left his mark in the theory of relativity was Hans Reichenbach.

A drawback of the neopositivists' approach to testing physical theories shows especially up when one attemps to experimentally test (at least in principle, on the level of so-called thought experiments) the equations of motion derived in a field theory, like for instance the general theory of relativity. Here are two elements that should be considered: the field, and the motion of the bodies which generate that field. A certain pecularity of the situation is due to the fact that there is no other way of measuring the field but by observing the motion of these bodies. Thus let us imagine that an astronomer wants to verify whether the consecutive events which form world lines of bodies he observed

1991 Mathematics Subject Classification: Primary 83C10.

The paper is in final form and no version of it will be published elsewhere. 
satisfy the general relativistic equations of motion. Following the traditional approach, if the verification is successful, the astronomer would say that he found yet another test of the theory of relativity. What would he however claim in case the verification has failed? That the theory of relativity is false? No, not at all. He would rather indicate that the bodies which were observed are not the only bodies in the system. That there are some additional bodies, maybe dark, that have modified the gravitational field.

The problem is even more accute, and relatively better suitable to an analysis, if one considers the motion of test bodies. In the early days of general relativity it was assumed that the world lines of test bodies were geodesics of the space-time manifold in question. And this assumption has been called the geodesic hypothesis. After the work of Infeld and Schild [1], which was examined recently more thoroughly in [2], it is known that the geodesity of these world lines is a conclusion which follows, in a sense of a certain limit, from the Einstein field equations of classical general relativity. It is however a convenient shortcut to label the corresponding statement, despite it is a theorem, still as 'the geodesic hypothesis'.

Now, what does it mean that the world line of a small body, a test body, is a geodesic line? The statement may be easily tested if on one side the world line of the body is known from observation, and the metric field is given by the theory on the other. But how can we test that a field predicted by the theory is the real one? Only by observing the motion of bodies. However, in order to draw a conclusion concerning the field, a law of motion must be assumed. How does this interplay of the two elements of the theory of relativity, of the motion and the field, agree with the view that the growth of science follows in consequence of inductive generalizations of experimental facts?

In this connection it should be indicated that since its very beginning the inductive approach to the theory of knowledge has been criticized from several positions. Especially appealing to contemporary scientists seems to be the critique expressed in The Logic of Scientific Discovery by Karl R. Popper, first in 1935, and then in his subsequent work. Popper proposed to replace the inductive program by another one, named the falsifiability of scientific statements and theories. A legitimate scientific statement should be according to Popper falsifiable. In a rather naive sense, it means that a scientific statement already in its formulation should contain a unique prescription indicating how it could possibly be invalidated by experimental tests. The aim, however, of the falsifiability program is not to raise doubt about the certitude of the roots of scientific theories. On the contrary, in accordance with this program, only those theories are certain which, firstly, satisfy the falsifiabilty requirement, and, secondly, will produce a negative response to all the experimental trials of falsifying them. Since the work of Popper, his program was widely discussed, criticized, and developed to a more sophisticated stage, cf. [3].

Returning to the geodesic problem, the interplay between the motion and field, mentioned above, seems to indicate that the geodesic hypothesis is not falsifiable. In the case, however, of a rich physical theory, like the theory of relativity together with its interpretational hull, one should be rather careful with expressing that type of judgement. The theory may offer enough possibilities to make constructions that permit one to view relations between basic theoretical concepts differently than they might initially look like from the point of view of their definitions alone. 
The objective of the present paper is to indicate that the seemingly vicious circle involving the relation between the motion and the field in attempts to show that the geodesic hypothesis is falsifiable, as it was described above, can be disentangled. The solution of the problem is based on theorems about the desynchronization of clocks which were obtained by the author in [4].

First, in Sections II - IV, the procedure, and its properties, of synchronization of clocks carried by families of observers is presented, and then in Sec. V, the conclusions that has been derived are used to solve the falsifiability problem of the geodesic hypothesis.

II. The locus of simultaneous events. The Einsteinian synchronization procedure of clocks carried by inertial observers in Minkowski space-time can be extended to the case of clocks of any mutually adjacent observers in an arbitrary space-time with Lorentzian signature, as it was, for instance, done in [5]. There an observer $O_{1}$ (Fig. 1)

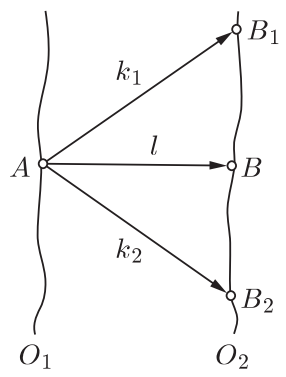

Fig. 1

sends from a space-time point $A$ a light signal to an infinitesimally close observer $\mathrm{O}_{2}$ and simultaneously receives at $A$ a light signal sent to him by $O_{2}$. The two signals are represented by the null vectors $k_{1}$ and $-k_{2}$, respectively, that are tangent to null geodesics connecting the timelike worldlines $O_{1}$ and $O_{2}$. The vectors $k_{1}$ and $k_{2}$ intersect the line $O_{2}$ at $B_{1}$ and $B_{2}$ correspondingly. The space-time point $B$ on the line $O_{2}$ chosen so that the proper time distances $d s_{B_{2} B}$ and $d s_{B B_{1}}$, measured by the clock carried by $O_{2}$, are equal to one another is by definition called simultaneous with the point $A$. Note that from the construction it follows that the vector $l$ which connects the points $A$ and $B$, in terms of the space-time metric $g_{\alpha \beta}$, is orthogonal at $A$ to the four-velocity $u$ of the observer $O_{1}$. This definition of simultaneity, unlike in the theory of special relativity, is limited to neighbouring world lines only. It can, however, still be used as a definition of the synchronization of origins of the time shown by proper clocks carried by two neighbouring observers in a general space-time. In [5] it is indicated, using only arguments of a qualitative type, that when we have a long sequence of observers then, starting from the first one, any next two neighbouring observers, who are infinitesimally close to each other, may, after having exchanged light signals, synchronize their clocks in the above meaning. The procedure of synchronization can thus be extended to observers who are no longer in an infinitesimal neighbourhood of one another. In the case, however, when the sequence forms a closed loop, the last of the observers who synchronized their clocks 
in the sequence will again be in an infinitesimal neighbourhood of the one from whom the synchronization procedure was started. These two observers will then in general find that their clocks are not synchronized, but a synchronization gap being equal to the difference of the proper times of their clocks will in general occur.

The situation just described can easily be put down in quantitative terms. The sequence of observers can be represented by a continuous one-parameter family of observers whose history in space-time is described by a string, i.e. by a two-dimensional timelike world sheet $\mathcal{S}$ given by the four equations

$$
x^{\alpha}=\xi^{\alpha}(s, \rho), \quad \alpha=0,1,2,3,
$$

where $s$ is the proper time parameter along each timelike world line which is specified by a value of the parameter $\rho$. The individual lines $x^{\alpha}=\xi^{\alpha}\left(s, \rho_{0}\right)$, for any $\rho=\rho_{0}=$ const of an admissible interval, are therefore world lines of observers who move, in general with an acceleration, in a given space-time. The four-velocity $u^{\alpha}$ of such an observer is

$$
u^{\alpha}=\frac{\partial \xi^{\alpha}}{\partial s}\left(s, \rho_{0}\right) \text {. }
$$

Any section of the string of observers $\mathcal{S}$ is a curve whose equations in space-time can be derived by substituting into Eqs. (2.1) in place of the variable $s$ a continuous function of the form $s=f(\rho)$. Thus a section of $\mathcal{S}$ is given in terms of a function $f$ by the equations

$$
x^{\alpha}=\xi^{\alpha}(f(\rho), \rho)=: \eta^{\alpha}(\rho) .
$$

The components of a vector $t$ tangent to this curve are equal to

$$
t^{\alpha}(\rho)=\frac{d \eta^{\alpha}}{d \rho}=\left[\frac{\partial \xi^{\alpha}}{\partial s} \frac{d f}{d \rho}+\frac{\partial \xi^{\alpha}}{\partial \rho}\right]_{s=f(\rho)}=\left[u^{\alpha} f^{\prime}+\frac{\partial \xi^{\alpha}}{\partial \rho}\right]_{s=f(\rho)} .
$$

Such a vector $t$, tangent to a curve of the form (2.3), can be used to define a connecting vector $l^{\alpha}=t^{\alpha} d \rho$ between the world lines $\xi^{\alpha}(s, \rho)$ and $\xi^{\alpha}(s, \rho+d \rho)$ at a point $A$ with the coordinates $\xi^{\alpha}(f(\rho), \rho)$. If we require that the clocks with these two world lines be synchronized, then in accordance with the construction discussed at the beginnig of this Section the connecting vector $l$ should be orthogonal to the four-velocity vector (2.2), i.e. $g_{\alpha \beta} u^{\alpha} l^{\beta}=0$. With the help of the relation $u^{\alpha} u_{\alpha}=1$, and Eqs. (2.4), this orthogonality condition can be brought to the form

$$
\frac{d f}{d \rho}=-g_{\alpha \beta}\left(\xi^{\mu}(f(\rho), \rho)\right) \frac{\partial \xi^{\alpha}}{\partial s}(f(\rho), \rho) \frac{\partial \xi^{\beta}}{\partial \rho}(f(\rho), \rho) .
$$

For a given family (2.1) of world lines and a given metric tensor field $g_{\alpha \beta}$ of the spacetime, relation (2.5) is an ordinary differential equation of first order for the function $f=f(\rho)$. Every solution of this equation permits us, by means of Eq. (2.3), to find a curve $x^{\alpha}=\eta^{\alpha}(\rho)$, which is a section of the family (2.1), whose tangent vector at any point is proportional to the connecting vector $l$ at that point. Since this vector connects pairs of events on adjacent world lines which are locally synchronized with each other, the whole curve to which it is tangent can be interpreted as a locus of events such that any "consecutive" pair of adjacent events along it is synchronized by a local procedure of exchanging light signals of the kind that is described at the beginning of this Section. In mathematical terminology the curve $x^{\alpha}=\eta^{\alpha}(\rho)$ is just an orthogonal trajectory of 
the family (2.1). In the physical context considered here, it will be called a locus of synchronized events. Equations (2.5) can now be considered as an exact, global definition of the synchronization of events belonging to different world lines of a one-parameter family of observers, whereas the local consideration presented in [5] may be treated only as its heuristic justification.

A solution $s=f(\rho)$ of Eq. (2.5), satisfying the initial condition

$$
f\left(\rho_{0}\right)=s_{0},
$$

determines a locus $x^{\alpha}=\eta_{0}^{\alpha}(\rho)=\xi^{\alpha}(f(\rho), \rho)$ of events which in the meaning discussed above are synchronized with the event $\xi^{\alpha}\left(s_{0}, \rho_{0}\right)$ lying on the world line $x^{\alpha}=\xi^{\alpha}\left(s, \rho_{0}\right)$ of the observer that is characterized by the value $\rho_{0}$ of the parameter labeling the family (2.1). The observer can then take this into account by simply resetting his clock so that the value of his proper time of the event of his meeting the locus $\eta_{0}^{\alpha}$ will also be equal to $s_{0}$. The fact which since the early days of relativity has been considered as interesting is of course not the mere resetting of clocks, but a consequence of it which occurs when the observers who one after another synchronize their clocks are situated along a closed loop in the three-space. If the synchronization procedure starts from the observer $\rho_{0}$ at the moment of his proper time $s=s_{0}$ and returns to him around the loop, he will find that the event which his nearest neighbour at the other end of the loop considers to be synchronized with $s_{0}$ will not be, after the synchronization which is "closing" the loop is performed, synchronized with the event $\xi^{\alpha}\left(s_{0}, \rho_{0}\right)$, but in general with a quite different event $\xi^{\alpha}\left(s_{1}, \rho_{0}\right)$ on his world line. This fact, amounting to a certain nonintegrability of the synchronization procedure along closed loops, is often called the desynchronization effect. The difference between the proper times $\Delta s=s_{1}-s_{0}$ of the two events will be called here the synchronization gap, cf. Fig. 2.

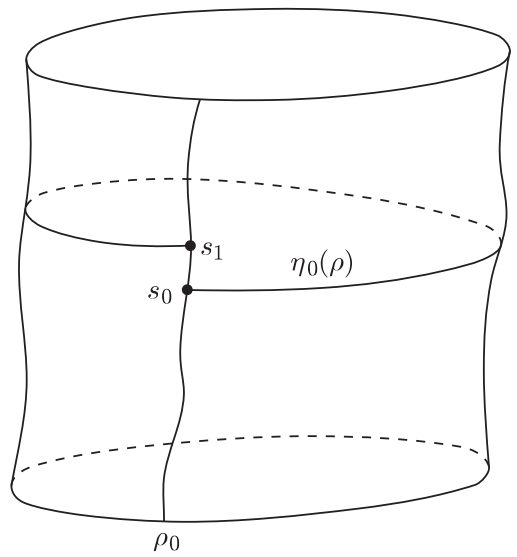

Fig. 2

Let us discuss this effect in terms of the space-time geometry. A string that in spacetime represents a spatially closed loop must have the topology of a three-cylinder. Such a string will be called closed. Analytically this means that the functions $\xi^{\alpha}$ must be periodic 
in their second argument:

$$
\xi^{\alpha}(s, \rho+2 \pi)=\xi^{\alpha}(s, \rho)
$$

(of course, after rescaling the parameter $\rho$, any period can be set to be equal to $2 \pi$ ). Thus, from a geometric point of view, the world line characterized by the parameter $\rho_{0}$ is exactly the same as that parametrized by $\rho_{0}+2 \pi$. Even though the functions $\xi^{\alpha}$ are periodic, the solution $s=f(\rho)$ of Eq. (2.5) is not necessarily a periodic one. As a result, the locus of synchronized events need not be a closed loop in space-time, and after returning to the initial observer $\rho_{0}$ it may suffer a jump. The value of this jump, measured in terms of the proper time of the observer $\rho_{0}$, is, in accordance with the definition given above, equal to the synchronization gap, and therefore

$$
\Delta s=f\left(\rho_{0}+2 \pi\right)-f\left(\rho_{0}\right) .
$$

Thus, to compute the synchronization gap of an observer $\rho_{0}$ belonging to a family of world lines described by Eqs. (2.1) which satisfy the periodicity condition (2.7), it is sufficient to solve first the differential equation (2.5) with the initial condition (2.6) and then to evaluate the difference (2.8).

ExAmple. Consider a family of world lines given in the Minkowski space-time by the equations

$$
\begin{aligned}
x^{0} & =\gamma s, \\
x^{1} & =\frac{v}{c} \gamma s \sin \rho+R \cos \rho, \\
x^{2} & =-\frac{v}{c} \gamma s \cos \rho+R \sin \rho, \\
x^{3} & =0,
\end{aligned}
$$

where

$$
\gamma^{-2}=1-\frac{v^{2}}{c^{2}}
$$

and the velocity of light $c$, the velocity $v<c$, and $R$ are constants; moreover $s,-\infty \leq$ $s \leq+\infty$, is the proper time parameter, and $\rho, 0 \leq \rho \leq 2 \pi$, is a parameter such that each of the lines labeled by $\rho=$ const is a straight timelike world line. The world sheet generated by the lines (2.9) is a hyperboloid of revolution

$$
\frac{v^{2}}{c^{2}}\left(x^{0}\right)^{2}-\left(x^{1}\right)^{2}-\left(x^{2}\right)^{2}=-R^{2}, \quad x^{3}=0 .
$$

One can easily write the differential equation (2.5) corresponding to the family of world lines (2.9) and to the Minkowski metric, and find its solution which satisfies the initial condition (2.6):

$$
f=\frac{v R \gamma}{c}\left(\rho-\rho_{0}\right)+s_{0}
$$

The analytic equations of the locus of synchronized events could now be obtained by substituting the r.h. side of Eq. (2.10) in place of the parameter $s$ in Eqs. (2.9). With the help of formula (2.8), one then obtains a nonvanishing synchronization gap equal to

$$
\Delta s=\frac{2 v R \gamma \pi}{c} .
$$


This example thus shows that even in Minkowski space-time there are families of observers who, though moving without an acceleration, will still observe a nonvanishing synchronization gap of inertial clocks carried by each of them.

Of course, in reality we do not have at our diposal continuously parametrized families of observers, but only a finite number of them. A locus of synchronized events is then a finite set of points. They are vertices of a broken straight line which is only an approximation of the locus resulting from the continuous formalism presented here. The accuracy of this approximation will depend on the number of observers involved.

III. Perfect synchronization. Let us consider an open string $\mathcal{S}$ of observers, i.e. a string for which the identifying condition (2.7) is not satisfied. Let us further select two observers from the family (2.1), an observer $O_{0}$ corresponding to the value $\rho_{0}$ of the parameter and $O^{\prime}$ characterized just by the value $\rho^{\prime}$, see Fig. 3 . Let $\eta_{0}$ be a locus of

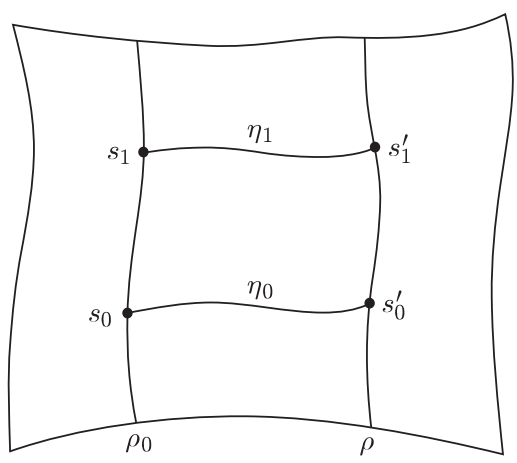

Fig. 3

synchronized events starting from an event on the world line of $O_{0}$ corresponding to the value $s_{0}$ of the proper time there. The observer $O^{\prime}$ intersects $\eta_{0}$ at a point corresponding to the value $s_{0}^{\prime}$ of his proper time. In accordance with the terminology accepted in [5], the clock carried by $O^{\prime}$ will be synchronized with that of $O_{0}$ if $O^{\prime}$ resets the origin of his clock so that $s_{0}^{\prime}=s_{0}$, which is always possible. In the discussion that was led in [5] there was however a very important point left aside. Assume that after a finite time interval at an event along $O_{0}$ 's world line corresponding to a value $s_{1}, s_{1}>s_{0}$, of $O_{0}$ 's proper time another locus of synchronized events $\eta_{1}$ is constructed. The observer $O^{\prime}$ meets $\eta_{1}$ at the time $s_{1}^{\prime}$ shown by his proper clock. The question that was not even posed is: can we expect that $s_{1}^{\prime}=s_{1}$ when at the moment of meeting $\eta_{0}$ the proper clock of $O^{\prime}$ was synchronized so that his proper time $s_{0}^{\prime}$ was numerically equal to the proper time $s_{0}$ of $O_{0}$. I propose to refer to the synchronization that allows a positive answer to the question above as to a perfect synchronization. Thus if the world lines of the two observers are parallel timelike straight lines in Minkowski space-time, the synchronization is certainly perfect. This is the case of the classical Einstein synchronization. We cannot however expect to attain a perfect synchronization in a more general situation of arbitrary families of observers of the form (2.1) immersed in a general space-time manifold with a Lorentzian signature. One of the conclusions arrived at in the following Section is a criterion stating for which 
families of observers immersed in a general space-time manifold a perfect synchronization is possible.

IV. Integral laws. An analysis of relations between geometric properties on a two dimensional string of observers $\mathcal{S}$, defined by Eqs. (2.1), is facilitated by a number of integral laws. In the first of these laws, which will be derived now, the string can be either open or closed. On the sheet $\mathcal{S}$ we have a vector field $u^{\alpha}$ whose value at any point of $\mathcal{S}$ is equal to the four-velocity $u^{\alpha}=\partial \xi^{\alpha} / \partial s$ of the observer at that space-time point. In terms of this vector field, the equations of motion of the observers can be written in the form

$$
u_{; \beta}^{\alpha} u^{\beta}=F^{\alpha},
$$

where the semicolon stands for the covariant derivative with respect to the metric connection of space-time, and $F^{\alpha}$ is the four-vector of force. Since $u^{\alpha} u_{\alpha}=1$, one finds that

$$
u_{\alpha ; \beta} u^{\alpha}=0,
$$

and the vectors $u$ and $F$ are orthogonal to each other. Subtracting Eqs. (4.1) and (4.2), one obtains

$$
u_{[\alpha, \beta]} u^{\beta}=\frac{1}{2} F_{\alpha},
$$

where the comma denotes ordinary, partial differentiation with respect to the coordinates, and the square brackets stand for antisymmetrization.

Consider now a two-dimensional compact region $\Sigma$ of the sheet $\mathcal{S}$, and take into account the following version of Stokes' theorem

$$
\int_{\Sigma} u_{[\beta, \alpha]} d \sigma^{\alpha \beta}=\oint_{\partial \Sigma} u_{\alpha} d x^{\alpha},
$$

where $\partial \Sigma$ denotes the boundary of $\Sigma$.

Let $t$ be a unit vector field on the sheet $\mathcal{S}$ orthogonal to the field $u$, tangent to $\mathcal{S}$, and pointing toward increasing values of the parameter $\rho$ in Eqs. (2.1). It is assumed that the inner orientation on $\mathcal{S}$ is defined by $u \wedge t$. The element of integration on the two-surface can then be written as

$$
d \sigma^{\alpha \beta}=2 u^{[\alpha} t^{\beta]} d s d \rho,
$$

and, due to Eqs. (4.3) and (4.5), the 1.h. side of Eq. (4.4) becomes

$$
\int_{\Sigma} u_{[\beta, \alpha]} d \sigma^{\alpha \beta}=\iint F_{\beta} t^{\beta} d s d \rho .
$$

Because of all the orthogonality relations which are valid here, the last integral can also be written in an intrinsic form

$$
\iint F_{\beta} t^{\beta} d s d \rho=\int_{\Sigma} F_{[\beta} u_{\alpha]} d \sigma^{\alpha \beta} .
$$

Comparing Eqs. (4.6) and (4.7), one obtains a relationship which could be called the first integral law for strings generated by world lines of particles (or observers) satisfying the 
equations of motion (4.3). It states that for any finite region $\Sigma \subset \mathcal{S}$

$$
\oint_{\partial \Sigma} u_{\alpha} d x^{\alpha}=\int_{\Sigma} F_{[\beta} u_{\alpha]} d \sigma^{\alpha \beta} .
$$

As was already said, Eq. (4.8) applies to a string $\mathcal{S}$ that can be either an open or a closed one, cf. Fig. 4.

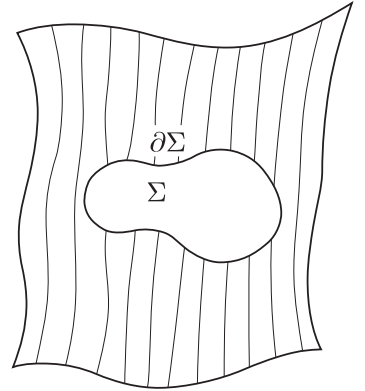

(a)

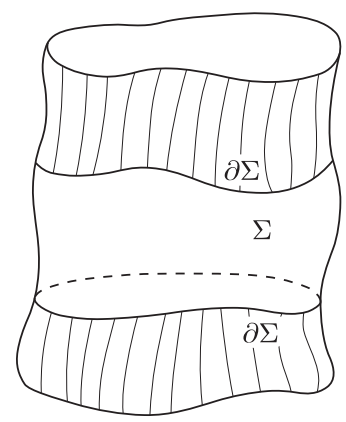

(b)

Fig. 4

Equation (4.8) can now be used for a derivation of the criterion mentioned at the end of Sec. III. To this end, let us chose for the region $\Sigma$ a "parallelogram" $\mathcal{P}$ formed by the arcs of the two world lines (2.1) that are characterized by $\rho=\rho_{0}$ and $\rho=\rho_{1}$, respectively, taken between the two loci $\eta_{0}(\rho)$ and $\eta_{1}(\rho)$ which were considered at the beginning of Sec. III, and by the two arcs of the loci between the lines $\rho=\rho_{0}$ and $\rho=\rho_{1}$, correspondingly. If the observer parametrized by $\rho_{1}$ resets his clock to $s=s_{0}$ when crossing the locus $\eta_{0}(\rho)$, then the vertices of the parallelogram will be parametrized by the pairs $\left(s_{0}, \rho_{0}\right),\left(s_{0}, \rho_{1}\right),\left(s, \rho_{1}\right)$, and $\left(s_{1}, \rho_{0}\right)$, where $s$ is the value of the proper time at which the observer $\rho_{1}$ crosses the locus $\eta_{1}(\rho)$, see Fig. 5 .

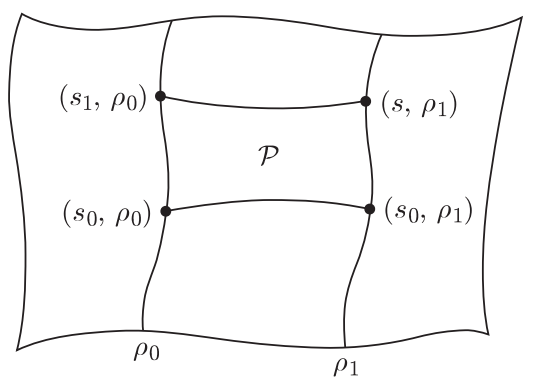

Fig. 5

The integral $\oint u_{\alpha} d x^{\alpha}$ along the boundary of the parallelogram will consist of four parts of which two, along the loci, will vanish, while the other two will be equal to 
$\left(s_{1}-s_{0}\right)$ and $\left(s-s_{0}\right)$, respectively. Thus from Eq. (4.8) one obtains that

$$
s_{1}-s=\int_{\mathcal{P}} F_{[\beta} u_{\alpha]} d \sigma^{\alpha \beta} .
$$

Equation (4.9) demonstrates that a perfect synchronization is not possible in general. It is attainable, however, if the integral in Eq. (4.9) vanishes for every parallelogram $\mathcal{P}$ that can be constructed on the sheet $\mathcal{S}$. This condition together with Eq. (4.1) lead to the the following criterion:

A perfect synchronization of proper clocks of a one-parameter family of observers is possible if and only if either the four-vector of force acting on observers is normal to the string $\mathcal{S}$ generated by them or all the world lines of the observers are geodesics with respect to the space-time geometry.

Another application of the first integral law (4.8) deals with a closed string of observers $\mathcal{S}$ which satisfy the periodicity condition (2.7). Let $\rho_{0}$ be an observer, selected by the value $\rho=\rho_{0}$ of the parameter from the family (2.1), who determines two loci (or more precisely speaking, two arcs of the loci each of which encircles the string exactly once) of synchronized events on $\mathcal{S}$ : one, $\eta_{0}$, satisfying the initial condition (2.6) and another, $\eta_{1}$, the condition $f\left(\rho_{0}\right)=s_{1}$, where $s_{1}>s_{0}$. The synchronization gaps defined by the two loci, respectively, are denoted by $\Delta s_{0}$ and $\Delta s_{1}$. Denote further by $\eta_{i+}, i=0,1$, the union of the $i$ th locus $\eta_{i}$ and of the arc of the world line $\rho_{0}$ (of "length" $\Delta s_{i}$ ) which joins the two ends of the locus. Let the region $\Sigma_{\ell}$, to which the theorem (4.8) will be applied, be the subset of the string $\mathcal{S}$ bounded by $\eta_{0+}$ and $\eta_{1+}$. Now, the integral on the r.h. side in Eq. (4.8) calculated along $\eta_{i+}$ will be equal to $\Delta s_{i}$, since the integral along the locus vanishes due to the orthogonality of $u$ and $t$. Therefore, Eq. (4.8) applied to the region $\Sigma_{\ell}$ becomes

$$
\Delta s_{1}-\Delta s_{0}=\int_{\Sigma_{\ell}} F_{[\beta} u_{\alpha]} d \sigma^{\alpha \beta} .
$$

The two sides of Eq. (4.10) can be divided by $s_{1}-s_{0}$, and one can pass with the whole equation to the limit $s_{1} \rightarrow s_{0}$. As a result, when Eq. (4.6) is taken into account, one obtains a relation which may be called the second integral law for strings generated by world lines of particles satisfying the equations of motion (4.3). It states that the proper time derivative of the synchronization gap evaluated along the world line of an observer measuring the gap is equal to the integral of the force vector along the locus $\eta_{0}$,

$$
\frac{d}{d s}(\Delta s)=\int_{\eta_{0}} F_{\alpha} d x^{\alpha} .
$$

In Eq. (4.11) the string of observers is fixed. For such a string, the integral on the r.h. side may vanish due to various reasons, leading to a conservation law of $\Delta s$. This happens, for instance, when the force $F$ is normal to the string or when it vanishes. Thus, the conservation of the synchronization gap $\Delta s$ along the world line along which it is measured is a necessary condition for all the world lines which generate the string (2.1) used for determining the gap to be geodesics. Equation (4.11) leads, however, also to 
another, more universal property, independent of a special shape or orientation in space of the family (2.1) of observers who measure a synchronization gap. This property can be formulated in terms of the following theorem.

THEOREM. Geodesics are the only world lines which will lead to a conserved synchronization gap for all kinds of closed strings which might be generated by the observers in order to determine the gap.

There are also two additional integral laws that apply to the synchronization of clocks carried by a one-parameter continuous family of observers in space-times of special and general relativity. They are reported in [4], and as not being relevant to the problem discussed now, they will not be quoted in this paper.

V. Conclusions. We are now prepared to solve the problem posed in the Introduction. In accordance with the results derived in Sec. IV, to falsify the geodesic hypothesis it is sufficient to measure the evolution of sychronization gaps formed by various closed, continuous families of freely falling test satellites. No knowledge of the metric field is required here. All that the observers in the satellites must be able to do is to send and receive light signals and to measure the proper time along their world lines. Thus every one of them must send a light signal to his nearest neighbour situated on a chosen side, say on the left, receive the reflected signal and take the event labelled by the mean time between the values of the emission and the reception of the reflected signal to be simultaneous with the event of reflecting the signal at the neighbouring world line. After the procedure is continued around the closed loop, the last observer in the row, being also the first one from whom the procedure was started, let us call him $O$, finds that the event he considers to be simultaneous with all the synchronized events on his left differs from the event from which the synchronization procedure was started, and the proper time that passed between these two events on his line is the synchronization gap. To find it, there is no necessity of performing the synchronization procedure on all the satellites simultaneously. It can be done afterwards by comparing the records in the satellites' logs. The observer $O$ should keep track of the behaviour of the synchronization gap in time, because its variability in time would, in accordance with the theorems just discussed, falsify the geodesic hypothesis. It is important that the falsification program be performed for various families and initial conditions. This would exclude the possibility of the existence of forces which, although describe deviations from geodesity, have no influence on the variability of the synchronization gap due to their accidental orthogonality to the two-surface spanned by the satellites, because of the vanishing of the l.h. side in Eq. (4.10) in such a case even for nongeodesic world lines.

As it has been already said, in practice we do not have continuous families of satellites at our disposal, but only a finite number of them. The continuous case is rather easy for a general discussion, like that in Sec. IV, whereas the discrete case requires a separate treatment for every set-up of the moving bodies, which additionally, in most cases, cannot be performed in an exact way. The continuous approach can thus be regarded as an approximation of a corresponding discrete case, and the quality of the approximation 
ought to be separately scrutinized in every particular case. We shall illustrate this point by means of a rather naive, but simple example.

EXAMPLE. Let us consider a family of observers whose world lines are circular spirals in Minkowski space-time for which Eqs. (2.1) take the form

$$
\begin{aligned}
x^{0} & =\gamma s, \\
x^{1} & =r \cos \left(\frac{\omega}{c} \gamma s+\rho\right), \\
x^{2} & =r \sin \left(\frac{\omega}{c} \gamma s+\rho\right), \\
x^{3} & =0,
\end{aligned}
$$

where $r$ and $\omega$ are constants, and

$$
\gamma^{-2}=1-\frac{\omega^{2} r^{2}}{c^{2}}
$$

The synchronization gap for these observers can be easily found as

$$
\Delta s=\frac{2 \pi \omega r^{2} \gamma}{c}
$$

provided the synchronization was performed clockwise, a minus sign would be produced in the other case.

If instead of the continuous family (5.1) one considers a set of $n$ bodies whose world lines are described by equations similar to (5.10) in which the continuous parameter $\rho$ must be now replaced by the discrete values $\rho_{i}=(2 \pi i) / n$ for $i=0,1, \ldots, n$, then the corresponding synchronization gap $\Delta s_{n}$ cannot be derived from the general formalism described above, but must be calculated directly from the world lines of the bodies. After some analytic geometry in Minkowski space, one can find that $\Delta s_{n}$ is given as the solution of the transcendental equation

$$
\Delta s_{n}=\frac{\omega r^{2} n}{\gamma c} \sin \frac{1}{n}\left(\frac{\omega \gamma}{c} \Delta s_{n}+2 \pi\right) .
$$

It is easy to see that in the limit of $n \rightarrow \infty,(5.2)$ is a solution of Eq. (5.1). For

\begin{tabular}{|c|c|c|c|c|c|c|c|c|c|c|}
\hline$n$ & 3 & 4 & 5 & 6 & 9 & 12 & 15 & 20 & 50 & 100 \\
\hline$\overline{\tau_{n} \cdot 10^{5}}$ & .375 & .577 & .686 & .749 & .834 & .865 & .880 & .891 & .904 & .906 \\
\hline
\end{tabular}
finite $n$, however, Eq. (5.3) can be solved only numerically. Thus in order to compare the difference between the two aproaches, one must assume some numerical values for the parameters. Let us take $r=4.225 \cdot 10^{7} \mathrm{~m}$, the value of the radius of the circular orbit of a geostationary satellite, and $\omega=7.272 \cdot 10^{-5} \mathrm{~s}^{-1}$, its angular velocity. Then the synchronization gap measured in seconds, $\tau=\Delta s / c$, takes the value $.906 \cdot 10^{-5} \mathrm{~s}$. The corresponding values of the gaps $\tau_{n}=\Delta s_{n} / c$ are given in the following table:

Thus first after the number of satellites is larger than twelve, one obtains here, within the accuracy of one significant digit, the same result as for $n \rightarrow \infty$. Thus only then the continuous approach can be considered as a reasonable approximation of the discrete one. The result may, of course, be quite different for other systems of bodies one would like to discuss. 
Acknowledgments. The research reported here has been supported by the Polish Research Program KBN, contract PB 898/P3/94/07, registration no 2 P302 11207 and the program BST-531/T.

\section{References}

[1] L. Infeld and A. Schild, Rev. Mod. Phys., 21, 408 (1949).

[2] J. M. Nevin, GRG, 27, 397 (1995).

[3] I. Lakatos, Falsification and the methodology of scientific research programmes, in Criticism and the Growth of Knowledge, I. Lakatos and A. Musgrave, eds., Cambridge University Press, Cambridge, 1974.

[4] S. L. Bażański, The desynchronization effect in relativity, in preparation.

[5] L. D. Landau and E. M. Lifshitz, Classical Theory of Fields (Addison-Wesley, Reading, Mass., 1971), 3rd edition, pp. 233-236. 